\title{
Los Planes de igualdad de oportunidades como instrumento de una política de cambio social
}

\author{
Juan José García Escribano \\ Universidad de Murcia \\ Inmaculada Concepción Sánchez Ruiz \\ Universidad de Murcia
}

Resumen

En numerosas ocasiones, desde la política se pretende alcanzar excelsos objetivos de cambio social y cultural relacionados con la consecución de la igualdad real entre mujeres y hombres, pero para ello es ineludible la institucionalización de organismos de igualdad en los diferentes niveles de gobierno y de instrumentos que posibiliten las acciones necesarias para la implementación de esas políticas. En el presente artículo se realiza un análisis de contenido de los Planes de igualdad desarrollados en los municipios de la Región de Murcia, con el objetivo de conocer la incidencia que tiene la legislación en igualdad sobre el desarrollo de Planes de igualdad en el ámbito municipal, así como si los mismos han servido como instrumento de políticas de cambio social.

\section{Palabras clave}

Planes de igualdad; cambio social; género; normativa; política

Códigos JEL: D78, I38, J16, J71, K38, Z18

\section{EQUALITY OPPORTUNITY PLANS AS AN INSTRUMENT OF A SOCIAL CHANGE POLICY}

\section{Abstract}

On many occasions, politics is intended to achieve lofty objectives of social and cultural change related to the achievement of real equality between women and men, but for this, the institutionalization of equality bodies at different levels of government and instruments is unavoidable. Those enable the actions necessary for the implementation of these policies. In this article, a content analysis of the equality plans developed in the municipalities of the Murcia Region is carried out, with the aim of knowing the impact that equality legislation has on the development of equality plans at the municipal level, as well as whether they have served as an instrument of social change policies.

Keywords

Equality plans; social change; gender; regulations; politics

JEL codes: D78, I38, J16, J71, K38, Z18

Fecha de recepción del original: 14 de febrero de 2021; versión definitiva: 24 de mayo de 2021.

Juan José García Escribano, Universidad de Murcia, España.

Tel.: +34 868884096; E-mail: escriba@um.es; ORCID ID: https://orcid.org/0000-0002-7216-5803.

Inmaculada Concepción Sánchez Ruiz, Universidad de Murcia, España.

Tel.: +34 86888 3000; E-mail: inmaculadaconcepcion.sanchez@um.es; ORCID ID: https://orcid.org/0000-0002-2180-4409. 


\section{Los Planes de igualdad de oportunidades como instrumento de una política de cambio social}

Juan José García Escribano

Universidad de Murcia

Inmaculada Concepción Sánchez Ruiz

Universidad de Murcia

1. Introducción

Que las instituciones gubernamentales se dediquen expresamente a la promoción de los derechos y la igualdad de las mujeres es un objeto de difícil comprensión en el marco habitual del análisis sociológico y politológico. El proceso de promoción pública de la igualdad de género permite apreciar el surgimiento de un nuevo sector de la acción pública que podría ser analizado desde la sociología de los movimientos sociales o desde el análisis de las políticas pública. Sin embargo, en la práctica, se vienen utilizando distintos marcos de análisis, que han conducido a hablar de "política pública feminista" (feminist policy) (Mazur, 2002), de "instituciones militantes o activistas" (contentious institutions) (Bereni y Revillard, 2011) y hasta de "feminismo de Estado" (State Feminism) (McBride y Mazur, 2010; Valiente, 2006 y 2013; Bustelo y Ortbals 2007; Bustelo 2014). Esta imprecisión respecto de los marcos analíticos viene a señalar la naturaleza híbrida del objeto de estudio ya que, en tanto se utilizan recursos públicos, se podría hablar de política pública, pero de una política pública que, haciéndose eco del propio activismo de la sociedad, se presenta con vocación de militancia y de cambio social.

En primer lugar, hay que resaltar que esta orientación transformadora no está presente en todas las políticas que oficialmente promueven los derechos y la igualdad de las mujeres. McBride y Mazur (2010) señalan que es necesario comprender el posicionamiento de las instituciones que promueven estas políticas en relación con otros debates (formación profesional, prostitución, paridad, etc.), ya que las mismas pueden, según la época y el contexto político, adoptar o no los objetivos de transformación de las relaciones sociales de sexo promovidos por los movimientos feministas en el ámbito de la sociedad civil.

Desde el discurso político se pone muchas veces el acento en la consecución de ambiciosos objetivos de transformación social y cultural en relación con la igualdad entre mujeres y hombres, pero para ello es necesario la institucionalización de organismos de igualdad en los dife- rentes niveles de gobierno y de instrumentos que posibiliten las acciones transversales necesarias para el desarrollo de políticas de igualdad: desde leyes a unidades de género y Planes de igualdad (Lombardo y León, 2014).

Estos instrumentos, y en concreto los Planes de igualdad, en tanto que materializan las intenciones, son los que se analizan en este artículo con el fin de interpretar las pretensiones de transformación y cambio social que guían las políticas de igualdad.

2. Instrumentos de acción pública relacionados con la igualdad, y especialmente los Planes de igualdad

La igualdad efectiva entre hombres y mujeres es un principio reconocido a nivel nacional, europeo e internacional en numerosos textos normativos. En el ámbito internacional, el origen del compromiso por la igualdad le corresponde principalmente a la Organización de las $\mathrm{Na}$ ciones Unidas (ONU) que, en la Carta de San Francisco del 26 de junio de 1945, se comprometía a "reafirmar la fe en los derechos fundamentales del hombre, en la dignidad y el valor de la persona humana, en la igualdad de derechos de hombres y mujeres, y de las naciones grandes y pequeñas" (ONU, 1945: 1). Del mismo modo, la ONU establece políticas que promueven la igualdad de oportunidades y aprueba las primeras iniciativas, entre las que destacan la Declaración sobre la Eliminación de la Discriminación contra la Mujer en 1967 y las Conferencias Mundiales de la Mujer que comienzan en 1975. En los últimos años, la igualdad de género se ha convertido en un asunto prioritario de la agenda pública internacional promovida por la ONU, como el tercer Objetivo de Desarrollo del Milenio y el quinto Objetivo de Desarrollo Sostenible.

La Unión Europea (UE) incorpora el principio de igualdad y no discriminación en el año 1957 con la firma del Tratado constitutivo de la Comunidad Económica Europea, conocido como Tratado de Roma, que en su artículo 119 
establece la "igualdad de retribución entre trabajadores y trabajadoras para un mismo trabajo". Con posterioridad, los Tratados de Maastricht en 1992, de Ámsterdam en 1997 y de Lisboa en 2007, así como la adopción de la Carta de los Derechos Fundamentales de la Unión Europea en 2000, han ido garantizando la igualdad entre mujeres y hombres y la puesta en marcha de políticas que permitan lograrla de manera efectiva. El Consejo de la UE, en su reunión de 7 de marzo de 2011, aprueba el Pacto Europeo por la Igualdad de Género (2011-2020) en el que se reconoce que"la igualdad entre mujeres y hombres constituye un valor fundamental de la Unión Europea y que las políticas de igualdad de género son vitales para el crecimiento económico, la prosperidad y la competitividad" (Diario Oficial de la Unión Europea, 2011: 12).

En el ámbito nacional, la Constitución Española de 1978 reconoce el principio de igualdad y no discriminación desde una doble perspectiva: el artículo 14 recoge la conocida como "igualdad formal", al establecer que "Ios españoles son iguales ante la ley, sin que pueda prevalecer discriminación alguna por razón de nacimiento, raza, sexo, religión, opinión o cualquier otra condición o circunstancia personal o social", mientras que el artículo 9.2 desarrolla lo que se conoce como "igualdad real", al señalar que "corresponde a los poderes públicos promover las condiciones para que la libertad y la igualdad del individuo y de los grupos en que se integra sean reales y efectivas".

La Constitución Española, al reconocer la igualdad "formal" y "real", no precisa que hombres y mujeres sean idénticos, sino que trata de conseguir que los derechos, responsabilidades y oportunidades de ambos no dependan del sexo con que hayan nacido (Moreno y Gil, 2018). Se garantiza el principio de igualdad de trato entre mujeres y hombres, que debería asegurar la ausencia de toda discriminación, directa o indirecta, por razón de sexo y la presencia equilibrada en la vida pública, extremo este último desarrollado por la Ley Orgánica 3/2007, de 22 de marzo, para la igualdad efectiva de mujeres y hombres (BOE, núm.71, de 23 de marzo de 2007), que en su Disposición adicional primera indica que: "se entenderá por composición equilibrada la presencia de mujeres y hombres de forma que, en el conjunto a que se refiere, las personas de cada sexo no superen el sesenta por ciento ni sean menos del cuarenta por ciento" (2007: 25).

A pesar de todo, si analizamos la realidad que nos circunda contemplamos numerosos ámbitos en los que se evidencia la desigualdad entre mujeres y hombres. Los datos de Eurostat muestran que la brecha salarial entre mujeres y hombres, es decir, la diferencia de los ingresos brutos por hora entre los trabajadores y las trabajadoras, en 2018 fue de un 13,9\%, dato que sitúa a España, respecto a veintisiete países de la UE, en la posición catorce (Eurostat, 2020). Por otro lado, se ha comprobado que el 48,6 $\%$ de las españolas trabajadoras con hijos menores de 12 años compatibilizan el trabajo a tiempo completo con la educación de los hijos, frente a 51,4\% de las mujeres que trabajan a tiempo completo y no tienen niños pequeños. A medida que se tienen más hijos, la tasa de empleo femenino disminuye en casi el 19 \% (Millán, Santos y Pérez, 2015: 221-222). Estos datos remiten a otro importante aspecto de la desigualdad: la conciliación y la corresponsabilidad, donde se constata que en 2019 un $26,2 \%$ de mujeres (de 25 a 54 años) empleadas con un hijo trabajaba a tiempo parcial, frente al 5,3\% de hombres (INE, 2020). Esta situación ha sido agravada por la pandemia de COVID-19, ya que el cierre en numerosos países de centros educativos ha planteado importantes desafíos a madres y padres por la carga adicional de producción doméstica, trascendiendo en problemas en los ámbitos de conciliación y corresponsabilidad.

Durante el periodo de confinamiento ocasionado por la crisis sanitaria de la Covid19, desde marzo a junio de 2020, la brecha de género en los cuidados se acentuó, lo que dificultó el desempeño de la actividad investigadora de las mujeres de los Organismos Públicos de Investigación. De las respuestas a un cuestionario online lanzado en julio, se desprende que el $48,4 \%$ de las mujeres se responsabilizaron de la limpieza del hogar y el 43,8 \% asumió las responsabilidades de cuidados durante el confinamiento, mientras que sólo el 18,9\% de los hombres se encargó de la limpieza y el 18,3 \% asumió las responsabilidades de cuidados (Unidad de Mujeres y Ciencia, 2020: 9).

Otro estudio sobre "Mujer y Trabajo en remoto durante la Covid19", realizado por Mireia las Heras y María Barraza (2020), profesoras del Centro de Trabajo y Familia del Instituto de Estudios Superiores de la Empresa (IESE), en el que se entrevistó a mujeres de España, Chile, México y Guatemala, pone de manifiesto que las mujeres llegaron a dedicar un $15 \%$ más de tiempo a labores de cuidados que los hombres y que tener menores en casa les ha dificultado la realización de su trabajo. Asimismo, las mujeres han tenido un $20 \%$ más de fatiga metal que los hombres y un $16 \%$ más de estrés, causado por la sobrecarga de trabajo de cuidados de menores o dependientes. En el caso de las madres solteras, estos porcentajes se llegan a incrementar hasta el $33 \%$ y $18 \%$ (Las Heras y Barraza, 2020).

Las políticas de igualdad puestas en marcha en los años 80 del siglo XX en Europa pretendían disminuir, entre otras, las desigualdades que todavía siguen produciéndose, tal como reflejan los datos anteriores. Su objetivo fundamental era promover medidas y establecer prácticas encaminadas a la superación de todo tipo de obstáculos que impidieran alcanzar la igualdad real entre mujeres y hombres.

La aplicación de estas políticas se realiza a través de diferentes instrumentos tales como, entre otros, el enfoque de género, la incorporación del principio de transversalidad o mainstreaming de género, las acciones positivas, presupuestos con perspectiva de género y los Planes de igualdad.

Según la Federación Española de Municipios y Provincias (2006), los Planes de igualdad de oportunidades entre mujeres y hombres, constituyen un marco referencial que determina los objetivos que se persiguen por parte de todos los agentes implicados para eliminar la desigualdad por razón de sexo, requiriendo el establecimiento de un seguimiento durante su periodo de vigencia. El plan de igualdad se considera "una herramienta fundamental en las políticas de género, cuyo propósito es abordar las dimensiones de la discriminación y desigualdad de género, tales como las estructurales, derivadas de la división sexual en espacios y trabajo y las culturales derivadas de la imposición de determinados patrones de interpretación de la 
realidad social como los roles y los estereotipos" (Sánchez, Avilés y García-Escribano, 2020: 133).

La implementación de los Planes de igualdad ha de tener en cuenta que la programación, el seguimiento y la evaluación forman un continuo que se retroalimenta entre sí. En cada espacio será preciso analizar la realidad en todos los sentidos, tanto las políticas de igualdad existentes, la situación de igualdad y desigualdad en ese territorio, como los ámbitos relacionados con el empleo, la vivienda, la salud y la educación, entre otros, en los que se considere necesario actuar; los recursos que se van a destinar; las resistencias que habrá que superar; las buenas prácticas que será interesante potenciar; el papel que tendrán los movimientos sociales, las asociaciones y la población en general; y un largo etcétera que adecuará la propuesta del plan al territorio en un momento social, económico y político determinado. Con la ejecución de un plan de igualdad se adquieren compromisos para cambiar la realidad de desigualdad entre mujeres y hombres que, si se cumplen, impulsará cambios en la realidad social.

3. Desarrollo normativo e implementación de Planes de igualdad en el ámbito nacional, autonómico y local

La Ley Orgánica 3/2007, de 22 de marzo, para la igualdad efectiva de mujeres y hombres (BOE, núm.71, de 23 de marzo de 2007), se aprueba en España con la finalidad de disminuir las desigualdades de género existentes y alcanzar la igualdad real. Esta Ley promueve combatir las manifestaciones de discriminación por razón de sexo y fomentar la igualdad real entre mujeres y hombres.

En el artículo 17, intitulado'Plan estratégico de igualdad de oportunidades', del capítulo I, se establece lo siguiente: "El Gobierno, en las materias que sean de la competencia del Estado, aprobará periódicamente un Plan Estratégico de Igualdad de Oportunidades, que incluirá medidas para alcanzar el objetivo de igualdad entre mujeres y hombres y eliminar la discriminación por razón de sexo" (Ibid.: 12615).

En cumplimiento de este artículo, a mediados del mes de diciembre del año 2007 el Consejo de Ministros del Gobierno de España aprueba el I Plan Estratégico de Igualdad de Oportunidades. En el año 2014, se implanta el II Plan Estratégico de Igualdad de Oportunidades, 2014-2016, que toma en consideración los principales instrumentos programáticos adoptados por la UE en esta materia, como la Estrategia Europea para la Igualdad entre Mujeres y Hombres 2010-2015 y la Estrategia Europa 2020. Actualmente, se encuentra vigente el Plan Estratégico 2018-2021, que responde al compromiso del Gobierno con la igualdad efectiva de mujeres y hombres y colabora en el desarrollo de los objetivos y metas planteados en la Agenda 2030 de desarrollo sostenible, en relación con la igualdad de género y el empoderamiento de mujeres y niñas.
Con la finalidad de alcanzar la igualdad real y efectiva, la Ley 3/2007 impele de forma directa, en el artículo 64, a la aprobación y desarrollo de Planes de igualdad, al señalar que:

El Gobierno aprobará, al inicio de cada legislatura, un Plan para la lgualdad entre mujeres y hombres en la Administración General del Estado y en los organismos públicos vinculados o dependientes de ella. El Plan establecerá los objetivos a alcanzar en materia de promoción de la igualdad de trato y oportunidades en el empleo público, así como las estrategias o medidas a adoptar para su consecución. El Plan será objeto de negociación, y en su caso acuerdo, con la representación legal de los empleados públicos en la forma que se determine en la legislación sobre negociación colectiva en la Administración Pública y su cumplimiento será evaluado anualmente por el Consejo de Ministros (Ibid.:12622)

En 2019, al considerar que los resultados obtenidos en materia de igualdad de trato y oportunidades entre mujeres y hombres en el ámbito del empleo y de la ocupación habían sido más bien discretos o, incluso, insignificantes, se aprueba una modificación de la Ley Orgánica 3/2007 mediante el Real Decreto-ley 6/2019, de 1 de marzo, de medidas urgentes para garantía de la igualdad de trato y de oportunidades entre mujeres y hombres en el empleo y la ocupación (BOE, núm. 57, de 7 de marzo de 2019). La finalidad de éste era disminuir las situaciones de discriminación y de desigualdad que se entreveían a través de los datos de la brecha salarial y fomentar medidas para la implantación de Planes de igualdad en el ámbito de las empresas.

Recientemente ha sido aprobado el Real Decreto 901/2020, de 13 de octubre, por el que se regulan los Planes de igualdad y su registro y se modifica el Real Decreto 713/2010, de 28 de mayo, sobre registro y depósito de convenios y acuerdos colectivos de trabajo ( $\mathrm{BOE}$, núm. 272 , de 14 de octubre de 2020). Esta norma desarrolla el artículo 46.6 de la Ley Orgánica 3/2007 en relación con las materias y temas a las que este hace referencia, junto con otros aspectos para mejorar la eficacia de los Planes de igualdad y para incrementar la seguridad jurídica. En el artículo 5, intitulado 'Procedimiento de negociación de los Planes de igualdad", se aclara quiénes participarán en la comisión negociadora, y en los artículos 7 y siguientes los contenidos de los Planes de igualdad, creándose en el artículo 11 el Registro de Planes de igualdad de las empresas.

Si perjuicio de que antes de la aprobación de la Ley Orgánica 3/2007, de 22 de marzo, para la igualdad efectiva de mujeres y hombres, se habían aprobado algunas normativas autonómicas en relación con la materia, es tras su aprobación cuando las distintas Comunidades Autónomas comienzan la promulgación de las siguientes leyes:

- Ley $7 / 2007$, de 4 de abril, para la igualdad entre mujeres y hombres y de protección contra la violencia de género en la Región de Murcia.

- Ley 12/2007, de 26 de noviembre, para la promoción de la igualdad de género en Andalucía. 
- Ley $1 / 2010$, de 26 de febrero, canaria de igualdad entre mujeres y hombres de las Islas Canarias.

- Ley 12/2010, de 18 de noviembre, de igualdad entre mujeres y hombres de Castilla-La Mancha.

- Ley 2/2011, de 11 de marzo, para la igualdad de mujeres y hombres y la erradicación de la violencia de género del Principado de Asturias.

- Ley 8/2011, de 23 de marzo, de Igualdad entre mujeres y hombres y contra la violencia de género en Extremadura

- Ley 17/2015, de 21 de julio, de igualdad efectiva de mujeres y hombres de Cataluña.

- Ley 11/2016, de 28 de julio, de igualdad de mujeres y hombres de las Islas Baleares.

Apenas unos días después de la aprobación de la Ley Orgánica 3/2007, la Asamblea Regional de Murcia promulga la Ley $7 / 2007$, de 4 de abril, para la igualdad entre mujeres y hombres y de protección contra la violencia de género en la Región de Murcia (BORM, núm. 91, de 21 de abril de 2008).

El artículo 11 de la Ley murciana 7/2007, intitulado'Planes de igualdad de oportunidades entre mujeres y hombres', señala que:

El Gobierno Regional aprobará cada legislatura un plan general que recoja de forma coordinada y global las líneas de intervención y directrices que deben orientar la actividad de los poderes públicos murcianos en materia de igualdad de mujeres y hombres. En la elaboración de dicho plan el Gobierno facilitará la participación del resto de administraciones públicas de la Región de Murcia y su cumplimiento será objeto de una adecuada evaluación. (2008: 31922)

Por su parte, el artículo 15, intitulado 'Planes municipales de igualdad de oportunidades entre mujeres y hombres', estipula que (Ibid.: 31923):

1. Las administraciones locales determinarán como objetivo prioritario la elaboración y aprobación de sus respectivos planes municipales de igualdad de oportunidades, que tendrán un carácter integral, y fijarán de forma coordinada y global las líneas de intervención y las directrices que deben orientar la actividad de los poderes públicos locales en materia de igualdad de oportunidades y lucha contra la violencia de género.

2. Los planes contendrán cláusulas de evaluación y seguimiento sobre la ejecución de éstos, alcance de las medidas diseñadas o grado de consecución de los objetivos previstos.

Los primeros Planes de igualdad se pusieron en marcha en la Comunidad Autónoma de la Región de Murcia en 1993. De los cuatro primeros Planes de igualdad implantados desde 1993 hasta 2005 se dispone una información limitada, ya que no están accesibles para poder consultarlos. Actualmente, los datos más detallados de la Comunidad Autónoma de la Región de Murciase muestran en el portal web oficial de Igualdad y Prevención de la Violencia de Género, desprendiéndose de ellos los siguientes hitos:
- El I Plan de igualdad de oportunidades para Mujeres se desarrolla en el periodo 1993-1995, implementándose por la Dirección General de Mujer.

- La Dirección General de Mujer, Juventud y Familia, pone en marcha el II Plan de igualdad de oportunidades entre hombres y mujeres, que se desarrolla en el período 1997-2000.

- En el año 2004, el Instituto de la Mujer de la Región de Murcia implanta el III Plan de igualdad de oportunidades entre hombres y mujeres para el periodo 20042005.

- El IV Plan de igualdad de oportunidades entre hombres y mujeres 2009-2011es impulsado por el Instituto de la Mujer.

- Actualmente, se encuentra en proceso de desarrollo el Plan Estratégico Regional de Igualdad de Oportunidades entre Mujeres y Hombres 2016-2020.

4. Contenido de los Planes de igualdad: áreas que favorecen el cambio

El objetivo principal del análisis de contenido de los Planes de igualdad desarrollados en los municipios de la Región de Murcia ha sido analizar la incidencia de la aprobación de normativas sobre igualdad entre mujeres y hombres en el desarrollo de Planes en el ámbito municipal, a fin de constatar si los mismos han servido como instrumento de políticas de cambio social.

Esta revisión documental ha permitido identificar el objeto de estudio de cada Plan de igualdad, construir premisas de partida y consolidar los elementos para establecer relaciones de semejanza o diferencia entre ellos.

Del análisis del contenido de los Planes de igualdad desarrollados en la Región de Murcia se desprende que los mismos han podido ser un instrumento de una política de cambio social, pero para aclarar esta afirmación es necesario identificar qué áreas pueden favorecer el cambio o, por el contrario, limitarlo.

Entendiendo, que "hay cambio social cuando las relaciones propias y específicas de una entidad social se forman con cualidades distintivas que difieren de las precedentes" (Donati, 1993: 42), en el campo que nos ocupa, se produciría cuando se modificara la estructura de la sociedad respecto de su sistema de normas, creencias, valores y políticas, de tal forma que se orientaran a la igualdad de oportunidades entre mujeres y hombres. Este cambio se podría ver favorecido por la implantación de Planes de igualdad que actúen sobre las áreas, recogidas en el Índice Sintético para la valoración de Planes de igualdad de oportunidades entre hombres y mujeres (2020), que se especifican a continuación:

En primer lugar, el área de Conciliación y Corresponsabilidad, cuya finalidad es propiciar un enfoque integrador 
y sin distinción de género respecto a las responsabilidades en el ámbito familiar, laboral y personal. En este caso la conciliación es entendida como la participación equilibrada entre mujeres y hombre en la vida familiar y en el mercado de trabajo a través de la reestructuración y reorganización de los sistemas laboral, educativo y de recursos. Por su parte, la corresponsabilidad es concebida como la responsabilidad compartida de todas las personas, y no exclusivamente como un deber social del sexo femenino. Algunas de las medidas que favorecen el cambio social y que incluyen los Planes de igualdad son:

- Garantizar servicios de conciliación de la vida laboral, familiar y personal.

- Promover campañas de información y sensibilización orientadas a concienciar a la ciudadanía de los beneficios de la conciliación, la responsabilidad equitativa y la igualdad de oportunidades entre mujeres y hombres. - Impulsar la formación sobre igualdad de oportunidades en el ámbito familiar respecto al reparto de tareas familiares y de cuidados, dirigido a todos los sectores de la población.

A continuación, se encuentra el área de Representación, Gobernanza y Participación, que surge de la necesidad de conseguir la composición equilibrada de la presencia de mujeres y hombres en los diferentes ámbitos de la sociedad. En los Planes de igualdades percibida como la participación ciudadana y de los colectivos que hay en la comunidad. Estos están representados a todos los niveles y son partícipes de las decisiones mediante su implicación y opinión. Otros aspectos destacables a tener en cuenta son los presupuestos de género participativos y la equiparación de la mujer en puestos directivos y de toma de decisiones. Entre las medidas que se propician se puede destacar:

- Fomentar los procesos participativos, los espacios y los canales desde los cuales cualquier persona que lo desee pueda cooperar en la planificación, gestión y evaluación de las políticas de igualdad.

- Incrementar la participación de las mujeres en la vida social, política, económica y cultural.

- Favorecer la presencia, en igualdad real de condiciones entre mujeres y hombres, en los puestos en los que se toman las decisiones.

El área Empleo, hace referencia al impulso para la incorporación y promoción de las mujeres en el mercado laboral. De igual modo, alude a las condiciones desiguales de acceso de mujeres y hombres y a la implantación de medidas que contribuyan a disminuir este tipo de situaciones. Se considera necesario establecer criterios que fomenten la prevención de desigualdades como el acoso sexual, la brecha salarial, el techo de cristal o cualquier otra discriminación laboral por cuestión de sexo. Algunas de las principales medidas respecto a esta área son: - Aplicar medidas para disminuir la brecha salarial entre
mujeres y hombres.
- Desarrollar actuaciones para reducir la segregación laboral vertical y horizontal.

- Disminuir la brecha de género en el acceso a la formación y al uso de las tecnologías de la información y la comunicación.

- Sensibilizar e informar sobre igualdad de oportunidades entre mujeres y hombres en el mundo laboral.

- Ejecutar protocolos de actuación para casos de discriminación laboral.

De igual forma, el cambio social puede verse condicionado por algunas áreas que mantienen un excesivo peso del patriarcado en la sociedad, como son:

En primer lugar, el área de Violencia de Género. Según las estadísticas oficiales en España fueron asesinadas 45 mujeres en el año 2020 (Ministerio de la Presidencia, Relación con las Cortes e Igualdad, 2020), siendo estas cifras el resultado de un sistema patriarcal que ha perpetuado un orden jerarquizado y desigual entre mujeres y hombres, en el que se expone la violencia como un mecanismo de control social de la mujer que sirve para reproducir y mantener el statu quo de la dominación masculina. De este modo, se interiorizan una serie de mitos que continúan propiciando discriminaciones entre mujeres y hombres, y fomentando situaciones de desigualdad, como son:

- El poder, la dominación, la competencia y el control son esenciales como prueba de masculinidad.

- La vulnerabilidad, los sentimientos y las emociones en el hombre son signos de feminidad y deben ser evitados.

- Asignación de papeles y normas sociales diferenciados en razón del género.

Si se considera los Planes de igualdad como instrumento de una política de cambio social, se pueden introducir medidas para disminuir la violencia de género, tales como:

- Impulsar campañas de sensibilización y prevención de la violencia de género dirigidas a toda la ciudadanía y grupos específicos de población.

- Garantizar e incrementar la seguridad y protección de las mujeres que se hallan en situación de violencia. Asimismo, consolidar y mejorar la atención integral a las mujeres en situación de violencia de género.

- Fomentar programas en el ámbito educativo para la prevención de la violencia de género.

- Promover la realización de talleres para el fomento de la autoestima y las habilidades sociales de las mujeres como medida de prevención de la violencia de género. - Desarrollar cursos de defensa personal para mujeres con el objetivo de proporcionar conocimientos y herramientas de autoprotección.

En segundo lugar, el área de Educación, donde se constata que resulta esencial introducir cambios en la educación de niños y niñas en las primeras etapas educativas para conseguir, desde la infancia, la interiorización de los valores fundamentales de la igualdad. De igual forma, es esencial potenciar la formación del personal docente en materia de igualdad de oportunidades y perspectiva de 
género. Se necesita un compromiso por parte de todos los agentes educativos y poner en práctica, una enseñanza igualitaria que repercuta en el desarrollo del alumnado desde la perspectiva de género. Algunas de las actuaciones preventivas que pueden promover positivamente el cambio social, con la finalidad de eliminar discriminaciones y concienciar sobre la igualdad de género entre mujeres y hombres son:

- Fomentar la educación y formación obligatoria en igualdad de género en todos los niveles educativos.

- Promover la coordinación de los centros educativos con el Centro de profesores y recursos para favorecer la formación del personal docente en materia de igualdad.

- Impulsar grupos de trabajo de igualdad educativa con el personal responsable de igualdad de los centros educativos.

- Coordinación con las personas responsables de igualdad en los diferentes Consejos escolares.

- Fomentar Protocolos de igualdad a nivel de centros educativos.

- Realizar campañas de sensibilización en materia de igualdad de género.

- Asumir que la igualdad es tarea de todos y todas.

Finalmente, el área de Visibilización y Sensibilización se entiende como el conjunto de acciones específicas orientadas a visibilizar la situación de discriminación que sufren las mujeres en la sociedad. Actualmente, los aspectos sociales, culturales e ideológicos están influyendo en esta área debido a la cultura patriarcal y a los gobiernos de ideología conservadora que son esencialmente antifeministas. Esta realidad promueve la no aceptación social de la necesidad de la igualdad entre mujeres y hombres, a la que se suma la falta de compromiso político y no priorización de los temas de igualdad. Todo ello genera, entre otras cosas, insuficiencia de recursos, trabas burocráticas para la implantación de medidas, falta de cualificación del personal responsable de las políticas de igualdad y ausencia de dotación presupuestaria. Las medidas más destacadas que pueden beneficiar positivamente el cambio social en esta área son:

\footnotetext{
- Preparar campañas preventivas específicas de sensibilización con perspectiva de género para distintos grupos poblacionales.

- Visibilizar las actividades implementadas mediante intercambios de buenas prácticas.

- Sensibilizar a los medios de comunicación en relación con la importancia de trabajar desde la perspectiva de género.

- Incorporar la perspectiva de género en todos los ámbitos de forma transversal, fomentando la igualdad de oportunidades entre mujeres y hombres.

- Difundir los Plan de igualdad de cada sector territorial a nivel nacional, autonómico y municipal.
}

En resumen, si se pretende conseguir la igualdad de derechos, responsabilidades y oportunidades de mujeres y hombres, de niñas y niños, todas las áreas mencionadas requieren de estrategias y medidas concretas para su incorporación e cumplimiento en los Planes de igualdad impulsados por las distintas administraciones, instituciones, universidades, empresas, sindicatos y organizaciones no gubernamentales.

5. Los cambios sociales generados por los Planes de igualdad de oportunidades

La política de igualdad es ciertamente una política de derechos, en el sentido de que pretende hacer realidad el artículo 2 de la Declaración Universal de Derechos Humanos cuando señala que todas las personas tienen los mismos derechos y libertades, sin hacer distinción, entre otras cosas, por sexo. Pero, para que este enunciado se pueda desarrollar objetivamente, la igualdad debe traducirse en oportunidades reales y efectivas, y para ello la política de igualdad ha de ser también una política con una dimensión simbólica, en el sentido de la necesidad de promover un cambio en las estructuras de los valores y las actitudes, en definitiva, un cambio cultural y social. Se trata de transformar la imagen de la mujer en la sociedad y la imagen que las mujeres tienen de sí mismas, en un enfoque de empoderamiento y transformación de su propio status. Se hace necesaria una metamorfosis en los esquemas culturales, donde la diferenciación entre los valores y las valoraciones de género se desprendan de las raíces biológicas de las que partieron.

Ciertamente en las distintas áreas señaladas como las más importantes que recogen los Planes de igualdad para favorecer el cambio, se han producido, como se señala a continuación, avances significativos en los últimos años, lo que no significa que se haya conseguido la igualdad real entre mujeres y hombres.

En el área de Conciliación y Corresponsabilidadse constata que, según la Encuesta de empleo del tiempo del INE, en 2002/2003 el tiempo dedicado al hogar y a la familia por las mujeres era de 4 horas y 24 minutos, mientras que en la última (2009-2010) este tiempo había descendido en 17 minutos en las mujeres, y los hombres habían aumentado en 24 minutos. Parece que la tendencia ha ido en la dirección de la igualdad entre mujeres y hombres, pero, según la Encuesta de Calidad de Vida -ECV- de Eurofund, de 2016, las diferencias entre el tiempo dedicado todavía son enormes: las mujeres dedican aproximadamente 2 horas más al día que los hombres al hogar y a la familia, mientras que al cuidado o educación de hijos las mujeres dedican una media de 5 horas y 26 minutos de dedicación diaria frente a las 3 horas y 17 minutos que dedican los hombres (ClosinGap, 2019).

En el área de Representación, Gobernanza y Participación, según los datos del Instituto de la Mujer y para la Igualdad de Oportunidades, también se observa una tendencia favorable a la igualdad que señalan los datos: en 
2003 el porcentaje de alcaldesas sobre el total de personas que ocupaban ese puesto en los Ayuntamientos españoles era del 12,6\%, mientras que en 2019 había ascendido al 21,7 \%. También había ascendido entre estos dos años el porcentaje de concejalas, que había pasado del 25,5\% en 2003 al 40,8 \% en 2019. Igualmente, el porcentaje de mujeres en el Gobierno de España y en el de las distintas Comunidades Autónomas también se ha ido incrementando en estos años: en el Gobierno de España emanado de la VII Legislatura en el año 2000 había un 17,7 \% de mujeres, mientras que en el Gobierno nacido de la XIV Legislatura en el año 2020 hay un 47,8 \%. Sin embargo, en otras parcelas de la sociedad esta tendencia al incremento de la participación de las mujeres no se visualiza tan nítidamente: en los puestos ejecutivos de las empresas del IBEX, según el Instituto Europeo para la lgualdad de género (EIGE), en 2012 había un 5,7 \% de mujeres y en 2019 un 16,2 \%, pero entre los CEO de esas empresas en 2012 había un 2,9\% de mujeres, es decir, una única mujer por 34 hombres y en 2019 seguía habiendo una sola mujer al frente de este selecto grupo de empresas. En 2003 había un 2,4 \% de mujeres presidiendo una Cámara Oficial de Comercio, Industria, Servicios y Navegación y en 2020 un 2,3 \%. Con el ánimo de no seguir aportando datos en la misma dirección que podrían hastiar al lector, se termina constatando que algo similar también sucede en los más altos órganos judiciales: entre los magistrados y magistradas del Tribunal Constitucional, en 2000 había un 8,3\% de mujeres, es decir, una magistrada y en 2020 el porcentaje había aumentado al $18,2 \%$, puesto que ahora eran dos magistradas, una de ellas Vicepresidenta, sobre un total de 11.

En el área de Empleo, según la Encuesta de Estructura Salarial del INE, en el año 2009 el salario anual más frecuente en las mujeres representó el $87,9 \%$ del salario más frecuente en los hombres, mientras que en el año 2018 se produjo un descenso de un poco más de cuatro puntos porcentuales, ya que el salario anual más frecuente en las mujeres (15.484,4 euros) representó solo el $83,8 \%$ del salario más frecuente en los hombres (18.470,8 euros). Si se consideran los salarios anuales con jornada a tiempo completo, el salario de la mujer representaba en el año 2018 el $89,5 \%$ del salario del hombre, mientras que en 2009 representaba el $86,3 \%$. En la jornada a tiempo parcial, el porcentaje era del 85,7 y en 2009 del $87,7 \%$. Por lo que respecta a la brecha salarial de género que, según la definición de Eurostat, es la diferencia entre el salario bruto por hora de los hombres y el de las mujeres, expresado como porcentaje del salario bruto por hora de los hombres, se constata que, según el tipo de jornada, en el año 2018 la brecha salarial de género (no ajustada a las características individuales) fue de 6,5 en la jornada a tiempo completo y de 20,6 en la jornada a tiempo parcial. En el año 2010 la brecha salarial en jornada a tiempo completo era de 10,2 y en jornada a tiempo parcial 34,6.

En el área de Violencia de Género, según la Macroencuesta de Violencia contra la Mujer 2019, realizada a instancia de la Delegación del Gobierno contra la Violencia de Género a una muestra de 9.568 mujeres representativa de la población femenina residente en España de 16 o más años, el 11,0\% de las mujeres ha sufrido violencia física de alguna pareja actual o pasada en algún momento de su vida. El 1,6\% de las mujeres que tienen pareja en la actualidad afirma haber sufrido violencia física de esta pareja. Entre las mujeres que han tenido parejas en el pasado, el 17,1 $\%$ ha sufrido violencia de alguna de estas parejas pasadas a lo largo de su vida. Estos porcentajes son casi idénticos a los que se obtuvieron en la macroencuesta de 2015, en la que se estimaba que el $13,7 \%$ de las mujeres residentes en España de 16 o más años había sufrido, a lo largo de la vida, violencia sexual por parte de parejas, exparejas o terceros (Delegación del Gobierno contra la Violencia de Género, 2020). La violencia de género es todavía una de las asignaturas pendientes de la sociedad española, que ve como cada año pierden la vida decenas de mujeres a manos de sus parejas actuales o pasadas $y$, aunque entre los años 2003 y 2020 las víctimas mortales han ascendido a 1076, desde 2010 se constata una tendencia decreciente en la cifra anual. En este cambio de tendencia, entre otras muchas cosas, debe haber incidido las constantes campañas de concienciación y prevención social propuestas en los distintos Planes de igualdad que se vienen sucediendo desde el año 2000 con la campaña "La violencia contra las mujeres nos duele a todos, nos duele a todas", que fue una campaña en la que se mostraba el problema, pero sin ofrecer ninguna solución, ni ayuda, ni mensajes esperanzadores. Desde 2006, inicia la campaña "Contra los malos tratos gana la ley", cuya finalidad era informar y sensibilizar sobre el cumplimiento y aplicación de la Ley Integral en la lucha contra la violencia de género. Asimismo, en el año 2007, continúa la campaña "A la Primera señal de malos tratos, llama, 016. No esperes a que la violencia de género deje esta señal" de difusión del servicio telefónico de información y asesoramiento jurídico en materia de violencia de género. En este momento, con la puesta en marcha de estos recursos, además de sensibilizar, se comienza a ofrecer soluciones reales a las mujeres víctimas de la violencia de género. La última campaña que utiliza el eslogan“La violencia machista la paramos unidas", surge como consecuencia de que el Ministerio de Igualdad, ante la pandemia, pone en marcha un Plan de contingencia contra la violencia de género, recogido en el Real Decreto Ley 12/2020, de medidas urgentes en materia de protección y asistencia a las víctimas de violencia de género. Como señalan Rodríguez y Robles (2016: 106) las primeras campañas se centraban en la violencia física, descuidando otros tipos de maltrato, siendo a partir del año 2009 cuando las campañas empiezan a contemplar los diferentes tipos de maltrato que sufren las mujeres y se dirigen a toda la sociedad solicitando su rechazo y actuación ante este problema.

En el área de Educación, si se tiene en cuenta que la escuela es uno de los ámbitos de socialización primordiales. Como señalan Alberdi y Rojas (2005), la prevención de todas las formas de violencia de género comienza por la educación en la igualdad, para lo que se hace imprescindible la coeducación, además de la introducción de programas y temas transversales dentro del currículum. La educación en igualdad pretende la eliminación de estereotipos sexistas en la enseñanza, a través de la implicación de toda la comunidad educativa (profesorado, personal no docente, alumnado, familias y sociedad en general).

El artículo 14 del Convenio del Consejo de Europa sobre prevención y lucha contra la violencia contra la mujer 
y la violencia doméstica, hecho en Estambul el 11 de mayo de 2011, y ratificado por España en 2014 (BOE, 137 de 6 de junio de 2014), obliga a los Estados a emprender "las acciones necesarias para incluir en los programas de estudios oficiales y a todos los niveles de enseñanza material didáctico sobre temas como la igualdad entre mujeres y hombres, los papeles no estereotipados de los géneros, el respeto mutuo, la solución no violenta de conflictos en las relaciones interpersonales, la violencia contra la mujer por razones de género, y el derecho a la integridad personal, adaptado a la fase de desarrollo de los alumnos". En septiembre de 2017 el Senado y el Congreso de los Diputados aprobaron por unanimidad el Informe de la Ponencia de Estudio para la elaboración de estrategias contra la violencia de género y el Documento refundido de medidas del Pacto de Estado en materia de Violencia de Género, el cual hace referencia a la especial importancia de educar en "valores igualitarios y la educación afectivo-sexual obligatoria en todos los niveles educativos, fomentando que los mismos se aborden de forma integral (aspectos fisiológicos y afectivo-emocionales)". Por último, en el Preámbulo de la reciente Ley Orgánica 3/2020, de 29 de diciembre, por la que se modifica la Ley Orgánica 2/2006, de 3 de mayo, de Educación, se señala que la misma "adopta un enfoque de igualdad de género a través de la coeducación y fomenta en todas las etapas el aprendizaje de la igualdad efectiva de mujeres y hombres, la prevención de la violencia de género y el respeto a la diversidad afectivo-sexual, introduciendo en educación secundaria la orientación educativa y profesional del alumnado con perspectiva inclusiva y no sexista".

Para terminar, en el área de Visibilización y Sensibilización, si bien los mayores esfuerzos han estado centrados en la sensibilización y prevención de la violencia de género, desde las distintas administraciones también se vienen promoviendo campañas y acciones que tienen como finalidad concienciar a la sociedad sobre la importancia de generar cambios en los valores sociales que promuevan la igualdad entre mujeres y hombres. Por ejemplo, el día 8 de marzo -Día Internacional de la Mujer- se ha convertido en una jornada en la que se celebran los avances en materia de igualdad y se reivindica a través de movilizaciones convocadas por el movimiento feminista lo mucho que todavía resta por hacer. Como señalan Papí y Cambronero (2011: 185), "las acciones de sensibilización en materia de género también se pueden enmarcar en lo que se ha venido llamando marketing social, que utiliza las técnicas propias del marketing orientadas al cambio de ideas (creencias, actitudes o valores) y/o prácticas (actos o conductas)". Las distintas administraciones (Ministerios, Comunidades Autónomas y Ayuntamientos) son las principales promotoras de las distintas campañas de sensibilización, pero no las únicas ya que, desde universidades, empresas, sindicatos, organizaciones no gubernamentales, etc., también se vienen desarrollando iniciativas que contribuyen a la visibilización del problema, aunque su impacto real esté muy mediatizado por otro tipo de intervenciones e, incluso, situaciones estructurales que condicionan claramente los cambios sociales.

\section{Conclusiones}

En términos de logros, parece evidente que los distintos Planes de igualdad desarrollados por administraciones, instituciones, organizaciones y empresas han contribuido, como instrumentos de una política de cambio social, a reducir las desigualdades entre mujeres y hombres en los distintos ámbitos en los que se han desarrollado, aunque no sea posible cuantificar con exactitud en qué medida estos cambios responden únicamente a su impulso y aplicación.

La legislación española actual es insuficiente para conseguir la igualdad real entre mujeres y hombres, ya que, marca las medidas necesarias, pero no garantiza su puesta en práctica. Si observamos el impulso de la institucionalización de las políticas de igualdad en los municipios de la Región de Murcia, tanto en la creación de estructuras como en la aprobación de planes, ha sido determinante la aplicación de la Ley de lgualdad 7/2007, de 4 de abril, para la igualdad entre mujeres y hombres y, protección contra la violencia de género en la Región de Murcia. Sin embargo, este paso, con el tiempo se ha ido diluyendo o estancando en muchas de las actuaciones de los gobierno regional y locales.

Los datos señalan que sigue resultando indispensable implementar servicios como escuelas y centros de conciliación de la vida laboral, familiar y personal; diseñar y llevar a cabo campañas específicas de sensibilización con perspectiva de género para distintos grupos poblacionales; desarrollar actividades específicas de fomento del acceso al empleo de mujeres; aumentar las medidas específicas para la introducción de programas educativos no sexistas y la implantación de actividades coeducativas como medio para contribuir a la eliminación de roles y estereotipos y desarrollar recursos preventivos destinados a la sensibilización respecto de la violencia de género, tanto a nivel escolar como para toda la sociedad. Es de consideración señalar, que hay que seguir desarrollando las medidas que recogen los Planes de igualdad, pues son necesarios más elementos complementarios que impulsen y garanticen políticas de igualdad eficaces y coherentes.

El fortalecimiento de políticas, acciones y prácticas conducentes a fomentar la igualdad de oportunidades entre mujeres y hombres en todos los ámbitos y la participación y el esfuerzo desarrollado por las distintas administraciones a la hora de integrar el principio de igualdad en su actividad, tanto interna como externa, pone de manifiesto que los Planes de igualdad de género son uno de los instrumentos principales para conseguir ese cambio de comportamientos que elimine las desigualdades, injusticias y discriminaciones de género y equipare las relaciones de poder entre mujeres y hombres; para conseguir, en definitiva, un cambio social que modifique la estructura más íntima de nuestra sociedad en lo que respecta a sus creencias, costumbres, valores y normas en relación con la igualdad de género. 
Bibliografía

ALBERDI, I. y ROJAS, L. (2005): Violencia: tolerancia cero. Barcelona, Fundación "La Caixa".

BERENI, L. y REVILLARD, A. (2011): Contentious Institutions: Rethinking the Movement/State Intersection, Paper presented at the second European Conference on Politics and Gender, Central European University, Budapest, January 13th- $\neg 15$ th 2011. https://annereviIlard.files.wordpress.com/2011/09/berenilrevillard2011contentiousinstitutions.pdf.

BUSTELO, M. (2014): "Three Decades of State Feminism and Gender Equality Policies in Multi-Governed Spain", Sex Roles, 74 (3-4), pp. 107-120. DOI 10.1007/s11199-014-0381-9.

BUSTELO, M. y ORTBALS, C. (2007): "The Evolution of Spanish State Feminism. A Fragmented Landscape", en J. Outshoorn y J. Kantola (eds.), Changing State Feminism. Basingstoke, Palgrave, pp. 201-223.

CLOSINGAP (2019): Coste de oportunidad de la brecha de género en la conciliación. https://closingap.com/wp-content/uploads/2020/11/ Informe_-Conciliacion_compressed.pdf.

DELEGACIÓN DEL GOBIERNO CONTRALA VIOLENCIA DE GÉNERO (2020): Macroencuesta de Violencia contra la Mujer 2019. Madrid, Ministerio de Igualdad. https://violenciagenero.igualdad.gob.es/violenciaEnCifras/macroencuesta2015/Macroencuesta2019/home.htm.

DONATI, P. (1993): “Pensamiento sociológico y cambio social: Hacia una teoría relacional", Revista Española de Investigaciones Sociológicas, 63, pp. 29-51.

EUROSTAT (2020): Salarios y costes laborales. https://ec.europa.eu/ eurostat/statisticsexplained/index.php?title=Wages_and_labour_ costs/es\&oldid=494293.

FEDERACIÓN ESPAÑOLA DE MUNICIPIOS Y PROVINCIAS (2006): Guía para Elaborar Planes Locales de Igualdad. http://femp.femp.es/files/566182-archivo/Guia\%20elaboracion\%20Planes\%20Locales\%20lgualdad.pdf

INE (2020): Mujeres y hombres en España. https://www.ine.es/ss/ Satellite?L=es_ES\&c=INESeccion_C\&cid $=1259925472720 \& p=1254$ 735110672\&pagename=ProductosYServicios\%2FPYSLayout.

LAS HERAS, M. y BARRAZA, M. (2020): Mujer y Trabajo en remoto durante la Covid19. Centro Trabajo y Familia del IESE. https://mireialasheras. com/wp-content/uploads/2020/07/infografias-teletrabajo-COVID19.pdf.

LOMBARDO, E. y LEÓN, M. (2014): "Políticas de igualdad de género y sociales en España: origen, desarrollo y desmantelamiento en un contexto de crisis económica", Investigaciones Feministas, 5, pp. 13-35.

MAZUR, A. G. (2002): Theorizing feminist policy. Oxford, Oxford University Press.

MCBRIDE, D. y MAZUR, A. (2010): The Politics of State Feminism. Innovation in Comparative Research. Philadelphia, Temple University Press.

MILLÁN, M. G.; SANTOS, M. P. y PÉREZ, L. M. (2015): “Análisis del mercado laboral femenino en España: evolución y factores socioeconómicos determinantes del empleo", Papeles de población, 21 (84), pp. 197225.

MINISTERIO DE LA PRESIDENCIA, RELACIONES CON LAS CORTES E IGUALDAD (2020): Mujeres víctimas mortales por violencia de género en España a manos de sus parejas o exparejas. https://violenciagenero. igualdad.gob.es/violenciaEnCifras/victimasMortales/fichaMujeres/ pdf/Vmortales_2020_15_06.pdf.

MORENO, C. y GIL, S. (2018): “Las mujeres en la Guardia Civil: aspectos normativos", Cuadernos de la Guardia Civil, 56, pp. 61-28.

ORGANIZACIÓN DE LAS NACIONES UNIDAS (1945): Carta de las Naciones Unidas. http://www.defensa.gob.es/Galerias/defensadocs/cartaONU-1945.pdf.
DIARIO OFICIAL DE LA UNIÓN EUROPEA (2011): "Pacto Europeo por la Igualdad de Género 2011-2020", C.155, de 25 de mayo de 2011, p. 12.

PAPÍ-GÁLVEZ, N. y CAMBRONERO-SAIZ, B. (2011): “Acciones públicas de sensibilización de género. El esfuerzo de la administración regional y local en comunicación publicitaria (1999-2007)", Pensar la Publicidad, 5 (2), pp. 181-203.

RODRÍGUEZ LÓPEZ, M. y ROBLES ÁLVAREZ, D. (2016): "La publicidad institucional en España: análisis de las campañas contra la violencia de género del Gobierno (2006-2015)", Revista de Comunicación Vivat Academia, 134, pp. 86-108.

SÁNCHEZ, I. C.; AVILÉS, M. y GARCÍA-ESCRIBANO, J. J. (2020): “La implantación de Planes de igualdad de Oportunidades entre Mujeres y Hombres en los municipios de la Región de Murcia (España)", Azarbe, Revista Internacional de Trabajo Social y Bienestar, 9, pp. 83-92. https://revistas.um.es/azarbe/article/view/436001.

SÁNCHEZ, I. C.; AVILÉS, M. y GARCÍA-ESCRIBANO, J. J. (2020): Índice Sintético para la valoración de Planes de igualdad de oportunidades entre hombres y mujeres. Registro General de la Propiedad Intelectual, Ministerio de Cultura y Deporte.

UNIDAD DE MUJERES Y CIENCIA (2020): Resultados del cuestionario sobre el impacto del confinamiento en el personal investigador, UMYC del Ministerio de Ciencia e Innovación. https://www.observatorioigualdadyempleo.es/download/resultados-del-cuestionariosobre-el-impacto-del-confinamiento-en-el-personal-investigador/.

VALIENTE, C. (2006): El feminismo de Estado en España: El Instituto de la Mujer (1983-2003). Valencia, Universitat de Vàlencia.

VALIENTE, C. (2013): "Gender equality policymaking in Spain (2008-11): Losing momentum", en B. N. Field y A. Botti (eds.), Politics and society in contemporary Spain: From Zapatero to Rajoy. New York, Palgrave Macmillan, pp. 179-195 